\title{
Clinical value of neurofilament and phospho-tau/tau ratio in the frontotemporal dementia spectrum
}

Lieke H.H. Meeter, MD, Everard G. Vijverberg, MD, Marta Del Campo, PhD, Annemieke J.M. Rozemuller, MD, Laura Donker Kaat, MD, Frank Jan de Jong, MD, Wiesje M. van der Flier, PhD, Charlotte E. Teunissen, PhD, John C. van Swieten, MD, and Yolande A.L. Pijnenburg, MD

Neurology ${ }^{\circledR}$ 2018;90:e1231-e1239. doi:10.1212/WNL.0000000000005261

\section{Abstract}

\section{Objective}

To examine the clinical value of neurofilament light chain (NfL) and the phospho-tau/total tau ratio ( $\mathrm{p} / \mathrm{t}$-tau) across the entire frontotemporal dementia (FTD) spectrum in a large, welldefined cohort.

\section{Methods}

CSF NfL and $\mathrm{p} / \mathrm{t}$-tau levels were studied in 361 patients with FTD: 179 behavioral variant FTD, 17 FTD with motor neuron disease (FTD-MND), 36 semantic variant primary progressive aphasia (PPA), 19 nonfluent variant PPA, 4 logopenic variant PPA (lvPPA), 42 corticobasal syndrome, and 64 progressive supranuclear palsy. Forty-five cognitively healthy controls were also included. Definite pathology was known in 68 patients (49 frontotemporal lobar degeneration [FTLD]-TDP, 18 FTLD-tau, 1 FTLD-FUS).

\section{Results}

NfL was higher in all diagnoses, except lvPPA $(n=4)$, than in controls, equally elevated in behavioral variant FTD, semantic variant PPA, nonfluent variant PPA, and corticobasal syndrome, and highest in FTD-MND. The $\mathrm{p} / \mathrm{t}$-tau was lower in all clinical groups, except lvPPA, than in controls and lowest in FTD-MND. NfL did not discriminate between TDP and tau pathology, while the $\mathrm{p} / \mathrm{t}$-tau ratio had a good specificity (76\%) and moderate sensitivity (67\%). Both high NfL and low $\mathrm{p} / \mathrm{t}$-tau were associated with poor survival (hazard ratio on tertiles 1.7 for NfL, 0.7 for $\mathrm{p} / \mathrm{t}$-tau).

\section{Conclusion}

NfL and p/t-tau similarly discriminated FTD from controls, but not between clinical subtypes, apart from FTD-MND. Both markers predicted survival and are promising monitoring biomarkers for clinical trials. Of note, $\mathrm{p} / \mathrm{t}$-tau, but not NfL, was specific to discriminate TDP from tau pathology in vivo.

\section{Classification of evidence}

This study provides Class III evidence that for patients with cognitive issues, CSF NfL and $\mathrm{p} / \mathrm{t}$-tau levels discriminate between those with and without FTD spectrum disorders.
Correspondence Dr. Meeter

h.meeter@erasmusmc.nl

\section{MORE ONLINE}

$\rightarrow$ Class of Evidence

Criteria for rating

therapeutic and diagnostic studies

NPub.org/coe 


\section{Glossary}

$\mathbf{A} \beta_{42}=\beta$-amyloid 1-42; $\mathbf{A D}=$ Alzheimer disease AUC = area under the curve; bvFTD = behavioral variant frontotemporal dementia; CBS = corticobasal syndrome; CDR = Clinical Dementia Rating; CDR-SB = Clinical Dementia Rating-Sum of Boxes; $\mathbf{C I}=$ confidence interval; $\mathbf{C V}=$ coefficient of variation; FAB = Frontal Assessment Battery; FTD = frontotemporal dementia; FTLD = frontotemporal lobar degeneration; FUS = fused in sarcoma; lvPPA = logopenic variant primary progressive aphasia; MMSE = Mini-Mental State Examination; MND = motor neuron disease; $\mathbf{N f L}=$ neurofilament light chain; $\mathbf{n f v P P A}=$ nonfluent variant primary progressive aphasia; PPA = primary progressive aphasia; $\mathbf{P S P}=$ progressive supranuclear palsy; p-tau $=$ phosphorylated tau; svPPA $=$ semantic variant primary progressive aphasia; TDP $=$ TAR DNA-binding protein 43 ; t-tau $=$ total tau.

Frontotemporal dementia (FTD) is a heterogeneous disease encompassing behavioral variant FTD (bvFTD) and the primary progressive aphasias (PPAs): the semantic variant (svPPA), the nonfluent variant (nfvPPA), and the logopenic variant (lvPPA). ${ }^{1,2}$ Motor neuron disease (MND), progressive supranuclear palsy (PSP), and corticobasal syndrome (CBS) are considered as part of the FTD spectrum. ${ }^{3}$ The underlying pathology, frontotemporal lobar degeneration (FTLD), is heterogeneous with tau (FTLD-tau), TAR DNAbinding protein 43 (FTLD-TDP), or fused in sarcoma inclusions (FTLD-FUS). ${ }^{4}$ Underlying pathology can only be predicted in genetic FTD: microtubule-associated protein tau (MAPT) mutations cause FTLD-tau, and progranulin (GRN) and chromosome 9 open reading frame 72 (C9orf72) mutations cause FTLD-TDP. ${ }^{5}$ The svPPA and FTD-MND are often associated with FTLD-TDP, and PSP with FTLD-tau, whereas underlying pathology can poorly be predicted in bvFTD. ${ }^{6}$

Disease-modifying therapies against FTD are currently under development, thus robust markers to track disease progression are essential. Neurofilament light chain (NfL), reflecting neuroaxonal damage, is a promising monitoring biomarker for FTD and other neurodegenerative diseases. ${ }^{7-12}$ For pathology-specific therapies, biomarkers predicting pathology are paramount, and lower phospho-tau 181 to total tau $(\mathrm{p} / \mathrm{t}$-tau) ratios were found in small series of patients with FTLD-TDP compared to FTLD-tau. ${ }^{10,13,14}$ However, both biomarkers have scarcely been validated and directly compared in large series across the entire FTD spectrum, which is needed before implementation in practice. In the current study, we compared the clinical value of $\mathrm{NfL}$ vs the $\mathrm{p} / \mathrm{t}$-tau ratio across all clinical and pathologic subtypes of the FTD spectrum.

\section{Methods}

\section{Participants}

From our previously described cohorts from the Erasmus Medical Center and the VU University Medical Center, ${ }^{15,16}$ we selected 361 patients based on the following criteria: (1) clinical diagnosis of bvFTD, nfvPPA, svPPA, FTD-MND, PSP, CBS, or lvPPA with a probable underlying FTLD ${ }^{1,2,17,18}$; and (2) CSF available for research. Patients with CSF results suggesting Alzheimer disease (AD) (low CSF $\beta$-amyloid 1-42 $\left[\mathrm{A} \beta_{42}\right]$ and high $\mathrm{p}$ - or $\mathrm{t}$-tau level, applying local laboratory standards) were not included, unless a definite FTD diagnosis was established $(n=3) .{ }^{1,19}$ As control group $(n=45)$, we used controls and participants with subjective memory complaints with normal CSF $A \beta_{42}$ levels (>550 pg/mL) from previous studies. ${ }^{9,10}$ A definite diagnosis was ascertained in 68 patients based on known FTD-causing mutations (genotyping was performed when family history was positive) or autopsyconfirmation: underlying FTLD-TDP pathology in 49 patients (12 GRN, 26 C9orf72 [of whom 7 underwent autopsy], 1 optineurin, 10 FTLD-TDP), FTLD-tau pathology in 18 patients (11 MAPT [of whom 1 underwent autopsy], 5 FTLD-tau, 1 CBD, and 1 PSP), and 1 patient with autopsyconfirmed FTLD-FUS. For subanalyses on suspected pathology, patients with svPPA and FTD-MND were added to the FTLD-TDP group and patients with PSP to the FTLDtau group. Concomitant $\mathrm{AD}$ pathology in autopsied patients was scored by an experienced neuropathologist (A.J.R.) as low ( $n=21$, ABC score "not" or "low") or high ( $n=4, A B C$ score "intermediate" or "high"). ${ }^{20}$ For 1 of 26 autopsied patients, insufficient information was available for this scoring.

Disease onset was defined as the time of first symptoms (e.g., first personality change or language difficulties) noted by a caregiver. Age at death was acquired by web-based consultation of the Dutch municipal personal records database (not available for one patient). The Mini-Mental State Examination (MMSE) was used to examine global cognition; the Frontal Assessment Battery (FAB) for executive function; and the Clinical Dementia Rating (CDR) scale, including Sum of Boxes (CDR-SB) if available, for disease severity.

\section{Standard protocol approvals, registrations, and patient consents}

All patients (or legal representatives) provided written informed consent, and this study was approved by the local ethics committees.

\section{CSF analyses}

CSF was collected and stored at $-80^{\circ} \mathrm{C}$ until analyses according to international consensus protocols. ${ }^{21}$ All measurements were performed blinded to clinical information and in one single center. CSF NfL was determined with ELISA of UmanDiagnostics (Umeå, Sweden) in duplicate, according to 
the manufacturer's instructions over 5 different batches. Mean intra-assay coefficient of variation $(\mathrm{CV})$ was $1.2 \%( \pm 1.2 \%$ SD), and interassay CV ranged from $6.1 \%$ to $16.7 \%$. Samples with too low volume for a duplicate $(n=9)$ were included in the analysis as overall intrasubject CV was low. CSF phospho$\operatorname{tau}_{181}(\mathrm{p}$-tau) and total tau ( $\mathrm{t}$-tau) were measured by commercial ELISAs (Innotest; Fujirebio, Ghent, Belgium) and remeasured when CV was $>15 \%$.

For controls, all 3 biomarkers were measured, but for some patients, insufficient CSF volume was available; NfL levels were available in 335 patients, the $\mathrm{p} / \mathrm{t}$-tau ratio in 352 patients, and both in 324 patients.

\section{Statistical analyses}

Statistical analyses were performed in SPSS 21.0 for Windows (IBM Corp., Armonk, NY) applying a significance level of $p<$ 0.05, and graphs were drafted with GraphPad Prism 7 (GraphPad Software, La Jolla, CA). Demographic data were compared by Kruskal-Wallis tests with post hoc Dunn tests (Bonferroni-corrected), or $\chi^{2}$ tests. Both CSF NfL and $\mathrm{p} / \mathrm{t}$-tau ratio were nonnormally distributed (Shapiro-Wilk test) and normalized after log transformation. Biomarker levels across (clinical or pathologic) groups were compared on log-transformed data with correction for age by analyses of covariance with post hoc Šidák tests. In case of missing data, patients were removed from respective subanalyses. Diagnostic performance was assessed by areas under the curve (AUCs) with 95\% confidence intervals (CIs) obtained by receiver operating characteristic analyses, with optimal cutoff levels at the highest Youden index. ${ }^{22}$ Diagnostic performance combining the 2 markers was analyzed by receiver operating characteristic analyses on probabilities obtained by binary logistic regression when comparing 2 groups (patients vs controls, TDP vs tau pathology) or multinomial logistic regression for different clinical diagnoses. Diagnostic performance was compared between the markers by the Hanley \& McNeil method in MedCalc (Ostend, Belgium). ${ }^{23}$ Biomarkers were correlated to clinical variables using Spearman correlation coefficient $\left(r_{s}\right)$. Survival was examined by KaplanMeier curves on NfL tertiles with living patients as censored data, and Cox regressions on tertiles or continuous biomarker levels, adjusted for age, sex, disease duration (time between onset and CSF collection), and presence of MND. Five-year survival rates were extracted from the Kaplan-Meier curves. Multivariate regression examined the following influencing factors in the association between logNfL levels (dependent variable) and log-transformed $\mathrm{p} / \mathrm{t}$-tau ratio: age, disease duration, CSF $A \beta_{42}$ as proxy for $A D$ copathology, and MND (covariates entered in second block).

\section{Classification of evidence}

This case-control study provides Class III evidence that patients across the entire FTD spectrum can be discriminated from healthy controls by high CSF NfL levels (sensitivity $79 \%$, specificity $89 \%, p<0.001$ ) or by low $\mathrm{p} / \mathrm{t}$-tau levels (sensitivity $73 \%$, specificity 93\%, $p<0.001$ ).

\section{Results}

\section{Patient characteristics}

Demographic characteristics of the 361 patients and 45 controls are displayed in table. Patients with CBS or PSP were older than controls, and patients with PSP were older than patients with bvFTD. Sex did not differ among the diagnostic groups. Age at onset, disease duration at CSF collection, and survival per clinical diagnosis are displayed in table. In total, 195 patients had deceased and their mean survival after CSF collection was $3.7( \pm 2.6)$ years. Survival did not differ between patients with definite TDP vs tau pathology $(p=0.44$, log-rank test).

\section{Discrimination of clinical diagnosis}

All clinical diagnoses, except for lvPPA $(n=4)$, had higher CSF NfL levels than controls (figure 1A; $p$ values and median differences are displayed in table e-1, links.lww.com/WNL/ A319), with the strongest elevation in patients with FTDMND. CSF NfL was lower in patients with PSP than in those with bvFTD. No differences were found among the other clinical subgroups.

The $\mathrm{p} / \mathrm{t}$-tau ratio mirrored the results of $\mathrm{NfL}$, as it was lower in all clinical diagnoses compared to controls, except for lvPPA $(n=4)$, and patients with FTD-MND had the lowest values (figure 1C; table e-2, links.lww.com/WNL/A319). In addition, patients with bvFTD had lower levels than patients with PSP. The decreased $\mathrm{p} / \mathrm{t}$-tau ratio in clinical patients compared to controls was driven by elevated t-tau levels in patients $(p<0.001$; for $\mathrm{p}$-tau: $p=0.52)$.

\section{Diagnostic performance: Clinical diagnosis}

To discriminate patients from controls, CSF NfL achieved an AUC of 0.87 (95\% CI $0.81-0.92, p<0.001$ ), with a sensitivity of $79 \%$ and specificity of $89 \%$ (cutoff $\geq 1,613 \mathrm{pg} / \mathrm{mL}$, table e-3 [links.lww.com/WNL/A319], including negative and positive predictive values). A low $\mathrm{p} / \mathrm{t}$-tau ratio showed a similar performance (AUC 0.86 [0.83-0.90], $p<0.001$, sensitivity 73\%, specificity $93 \%$ at ratio $\leq 0.153$; compared to AUC NfL: $p=0.74)$. Combining these 2 markers by logistic regression yielded a higher AUC of $0.91(0.88-0.95, p<0.001$, Nagelkerke $R^{2}=0.45$, sensitivity $80 \%$, specificity $93 \%$; compared to AUC NfL: $p=0.03$; compared to AUC ratio: $p<0.01$ ).

In classifying the different clinical diagnoses, both NfL and $\mathrm{p} / \mathrm{t}$-tau ratio were able to discriminate FTD-MND and PSP from bvFTD (all $p<0.001$ ), and combining the markers did not improve this classification.

\section{Discrimination of pathologic diagnosis}

When NfL levels were analyzed based on definite pathology, no difference between FTLD-tau and FTLD-TDP was found $(p=0.96$, figure 1B). However, when suspected pathologies were added in the analysis (i.e., PSP in tau group; svPPA and FTD-MND in TDP group), higher levels were observed in patients with suspected TDP than in patients with 
Table Participant characteristics

\begin{tabular}{|c|c|c|c|c|c|c|c|c|c|}
\hline & Controls & bvFTD & FTD-MND & SVPPA & nfvPPA & IvPPA & CBS & PSP & $p$ Value \\
\hline No. & 45 & 179 & 17 & 36 & 19 & 4 & 42 & 64 & \\
\hline Age at CSF collection, y & $60(53-65)$ & $61(55-67)$ & $63(56-69)$ & $62(58-65)$ & $62(52-66)$ & $64(51-69)$ & $65(60-73)^{a}$ & $66(62-70)^{b}$ & $<0.001$ \\
\hline $\begin{array}{l}\text { Sex, male (\% within } \\
\text { clinical group) }\end{array}$ & $26(58)$ & $101(56)$ & $9(53)$ & $19(53)$ & $9(47)$ & $1(25)$ & $28(67)$ & $28(44)$ & 0.35 \\
\hline Age at onset, y & - & $58(51-64)^{c}$ & $61(55-67)$ & $57(55-62)$ & $60(50-65)$ & $62(50-67)$ & $62(56-71)$ & $63(57-67)$ & 0.001 \\
\hline $\begin{array}{l}\text { Time between onset and } \\
\text { CSF collection, y }\end{array}$ & - & $2.5(1.5-4.6)$ & $1.3(0.7-2.1)^{d}$ & $2.7(1.9-5.0)$ & $2.1(1.2-3.2)$ & $1.9(1.6-2.9)$ & $2.2(1.2-3.3)$ & $2.2(1.5-3.7)$ & 0.009 \\
\hline $\begin{array}{l}\text { Survival after CSF collection } \\
\text { in deceased patients, }{ }^{e} y\end{array}$ & - & $3.9(2.0-5.9)$ & $1.0(0.5-1.2)$ & $5.3(4.5-8.9)$ & $6.2(1.9-8.2)$ & 3.2 & $1.8(1.2-3.4)$ & $3.0(1.9-4.0)$ & $<0.001$ \\
\hline CSF NfL available & 45 & 164 & 14 & 36 & 19 & 4 & 40 & 58 & \\
\hline p/t-tau ratio available & 45 & 174 & 16 & 34 & 19 & 4 & 42 & 63 & \\
\hline CSF NfL, pg/mL & $\begin{array}{l}974 \\
(616-1,357)\end{array}$ & $\begin{array}{l}3,168 \\
(1,752-4,818)\end{array}$ & $\begin{array}{l}19,232 \\
(10,094-27,016)\end{array}$ & $\begin{array}{l}3,151 \\
(1,906-4,802)\end{array}$ & $\begin{array}{l}2,345 \\
(1,956-2,957)\end{array}$ & $\begin{array}{l}1,731 \\
(1,181-2,472)\end{array}$ & $\begin{array}{l}2,664 \\
(1,715-4,158)\end{array}$ & $\begin{array}{l}1,907 \\
(1,474-2,755)\end{array}$ & $<0.001$ \\
\hline CSF phospho-tau $181, \mathrm{pg} / \mathrm{mL}$ & $44(37-56)$ & $42(34-54)^{f}$ & $43(33-50)$ & $40(36-53)$ & $51(31-66)$ & $54(33-73)$ & $47(38-57)^{f}$ & $37(28-44)$ & 0.01 \\
\hline CSF total tau, pg/mL & $243(197-308)$ & $342(271-467)^{g}$ & $488(322-623)^{g}$ & $333(265-454)^{g}$ & $361(205-442)$ & $427(246-583)$ & $336(246-446)^{g}$ & $237(176-315)$ & $<0.001$ \\
\hline CSF $\beta$-amyloid 1-42, pg/mL & $987(874-1,142)$ & $893(726-1,072)$ & $985(732-1,162)$ & $877(728-1,048)$ & $969(840-1,219)$ & $861(486-1,033)$ & $810(607-999)^{\mathrm{h}}$ & $826(665-1,003)^{\mathrm{h}}$ & 0.001 \\
\hline p/t-tau ratio & $0.18(0.17-0.20)$ & $0.12(0.10-0.15)$ & $0.09(0.07-0.10)$ & $0.12(0.11-0.15)$ & $0.14(0.12-0.17)$ & $0.13(0.12-0.14)$ & $0.13(0.11-0.16)$ & $0.15(0.13-0.17)$ & $<0.001$ \\
\hline
\end{tabular}

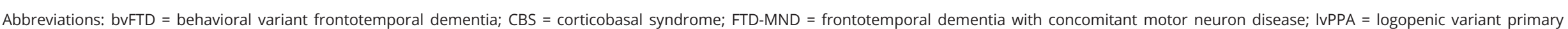

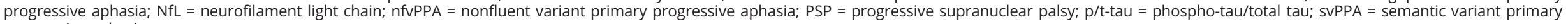
progressive aphasia.

Values are displayed as median (interquartile range); continuous variables are compared by Kruskal-Wallis tests.

a Older than controls.

b Older than controls and bvFTD.

'Younger than CBS and PSP.

d Shorter than SVPPA and bvFTD.

e Zero controls, 88 bvFTD, 13 FTD-MND, 18 svPPA, 6 nfvPPA, 1 IvPPA, 24 CBS, and 46 PSP patients were known to be deceased; CSF NfL was available in 178 and the p/t-tau ratio in 189 of them.

Higher than in patients with PSP.

${ }^{g}$ Higher than in controls and patients with PSP. 
Figure $1 \mathrm{NfL}$ and p/t-tau ratio levels by clinical diagnosis and by underlying pathology

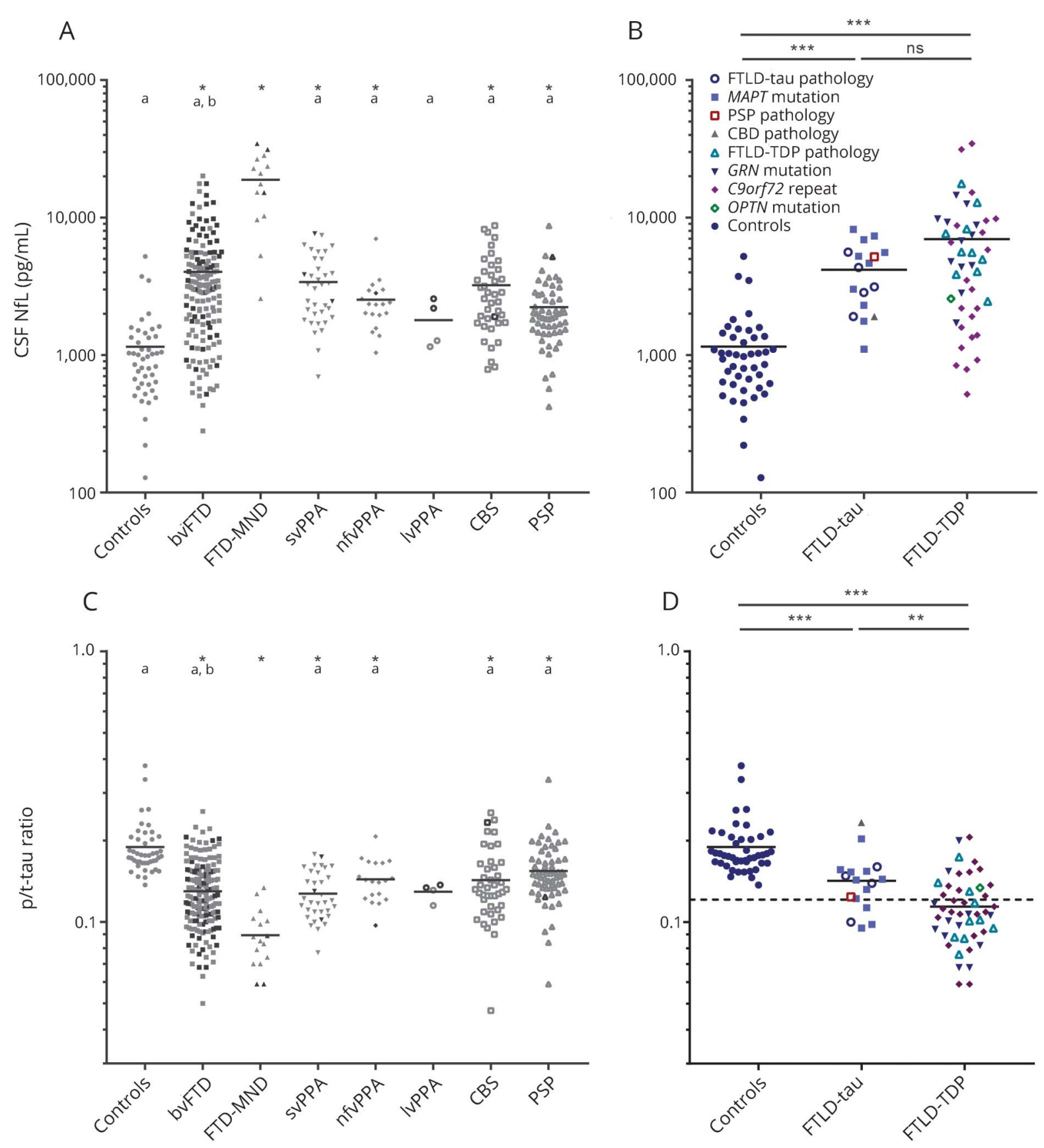

(A) NfL levels and (C) the p/t-tau ratio in clinical FTD subgroups; patients with confirmed underlying pathology are depicted in black. (B) NfL levels and (D) the p/t-tau ratio in patients with known underlying pathology based on autopsy-confirmed pathology (FTLD-tau = blue circles; PSP = red squares; CBD = gray filled upward triangles; FTLD-TDP = aqua upward triangles) or a known pathogenic mutation (MAPT = filled light-purple squares, GRN = filled blue downward triangles, C9orf72 repeat expansion = purple filled diamonds; OPTN = green diamonds); a low p/t-tau ratio (cut-off $\leq 0.121$, dashed line) discriminated FTLDTDP from FTLD-tau with a specificity of $76 \%$ and a sensitivity of $67 \%$. Horizontal lines represent means. $* \star \star p<0.001 ; * \star p<0.01 ; *(A)$ higher NfL or (C) lower p/t-tau ratio compared with controls; $a=(A)$ lower NfL or (C) higher p/t-tau ratio compared with FTD-MND, b = (A) higher NfL or (C) lower p/t-tau ratio compared with PSP. bVFTD = behavioral variant frontotemporal dementia; $C B D=$ corticobasal degeneration; $C B S=$ corticobasal syndrome; FTD-MND = frontotemporal dementia with concomitant motor neuron disease; FTLD-tau = frontotemporal lobar degeneration with tau inclusions; FTLD-TDP = frontotemporal lobar degeneration with TAR DNA-binding protein 43 inclusions; IvPPA = logopenic variant primary progressive aphasia; NfL = neurofilament light chain; nfvPPA = nonfluent variant primary progressive aphasia; ns = not significant; PSP = progressive supranuclear palsy; p/t-tau = phospho-tau/total tau; SVPPA = semantic variant primary progressive aphasia.

suspected tau $(p<0.001$, figure e-1A, links.lww.com/WNL/ A318). NfL levels were similar in autopsied patients with low vs high concomitant $\mathrm{AD}$ pathology $(p=0.83$, MannWhitney test).
The $\mathrm{p} / \mathrm{t}$-tau ratio was lower in definite or suspected TDP pathology than in those with definite or suspected tau pathology ( $p=0.005$ and $p<0.001$, respectively; figure $1 \mathrm{D}$ and figure e-1B, links.lww.com/WNL/A318). The decreased 
ratio in FTLD-TDP was driven by lower p-tau levels (median $38 \mathrm{pg} / \mathrm{mL}$ [interquartile range 29-44]) than in FTLD-tau (54 [42-67] $\mathrm{pg} / \mathrm{mL})$, while t-tau levels were similar (335 [252-448] pg/mL and 408 [310-522] pg/mL, respectively). The ratio did not differ between low and high concomitant $\mathrm{AD}$ pathology ( $p=0.78$, Mann-Whitney test $)$.

\section{Diagnostic performance: Pathologic diagnosis}

NfL did not differentiate between underlying TDP and tau pathology $(p=0.26)$, while the $\mathrm{p} / \mathrm{t}$-tau ratio did (AUC 0.73 [0.60-0.87], $p=0.005$, sensitivity $67 \%$, specificity $76 \%$ at ratio $\leq 0.121$; figure $1 \mathrm{D}$ and table e-3, links.lww.com/WNL/A319). Combining NfL and $\mathrm{p} / \mathrm{t}$-tau ratio did not improve the differentiation on underlying pathology (AUC 0.75 [0.62-0.88], $p=0.004 ; p=0.94$ vs AUC of ratio alone).

\section{Associations between biomarkers and clinical parameters}

$\mathrm{NfL}$ correlated moderately with t-tau $\left(r_{s}=0.51, p<0.001\right)$, weakly with p-tau $\left(r_{s}=0.13, p=0.02\right)$, and moderately with the $\mathrm{p} / \mathrm{t}$-tau ratio $\left(r_{s}=-0.62, p<0.001\right.$; figure 2$)$. This association between the $\mathrm{p} / \mathrm{t}$-tau ratio and NfL was influenced by MND and disease duration $\left(\Delta R^{2}=0.11, \mathrm{MND}: \beta=0.29\right.$, $p<0.001$; disease duration: $\beta=-0.09, p=0.04)$, but not by age or CSF $A \beta_{42}$. In definite patients, this association was not influenced by type of pathology (TDP vs tau, $p=0.25$ ).

NfL associated weakly with disease duration, MMSE, and $\mathrm{FAB}$, moderately with CDR-SB, but not with age, sex, or global CDR; the $\mathrm{p} / \mathrm{t}$-tau ratio associated weakly with age and disease duration, moderately with CDR-SB, but not with sex, MMSE, FAB, or global CDR (table e-4, links.lww.com/ WNL/A319). Patients with GRN mutation had higher NfL levels and lower $\mathrm{p} / \mathrm{t}$-tau ratios than those with C9orf72, $M A P T$, or no known mutations (table e-4).

\section{Prediction of survival}

Five-year survival was $73 \%$ in patients with low CSF NfL levels, 55\% in moderate levels, and 36\% in high levels (figure $3 \mathrm{~A}$; estimated hazard ratio 1.7 [95\% CI 1.3-2.1], $p<0.001$ ). After stratification on diagnosis, this association between high NfL levels and poor survival was confirmed in bvFTD $(p<$ $0.001)$, CBS $(p=0.001)$, and PSP $(p<0.001$; Cox regression on continuous NfL levels).

For the p/t-tau ratio, 5-year survival was $37 \%$ in patients with a low ratio, $56 \%$ in moderate ratios, and $63 \%$ in high ratios (figure 3B; estimated hazard ratio 0.70 [95\% CI 0.56-0.86], $p=0.001)$. Subanalyses by clinical diagnosis showed associations of lower $\mathrm{p} / \mathrm{t}$-tau ratios with a poorer survival in bvFTD and PSP $(p<0.001$ and $p=0.04$, respectively, Cox regression on continuous $\mathrm{p} / \mathrm{t}$-tau ratios).

\section{Discussion}

This study compared the clinical value of CSF biomarkers $\mathrm{NfL}$ and $\mathrm{p} / \mathrm{t}$-tau ratio in a large cohort of patients with FTD. We showed that both biomarkers (1) discriminate patients

Figure 2 Association between NfL and the p/t-tau ratio
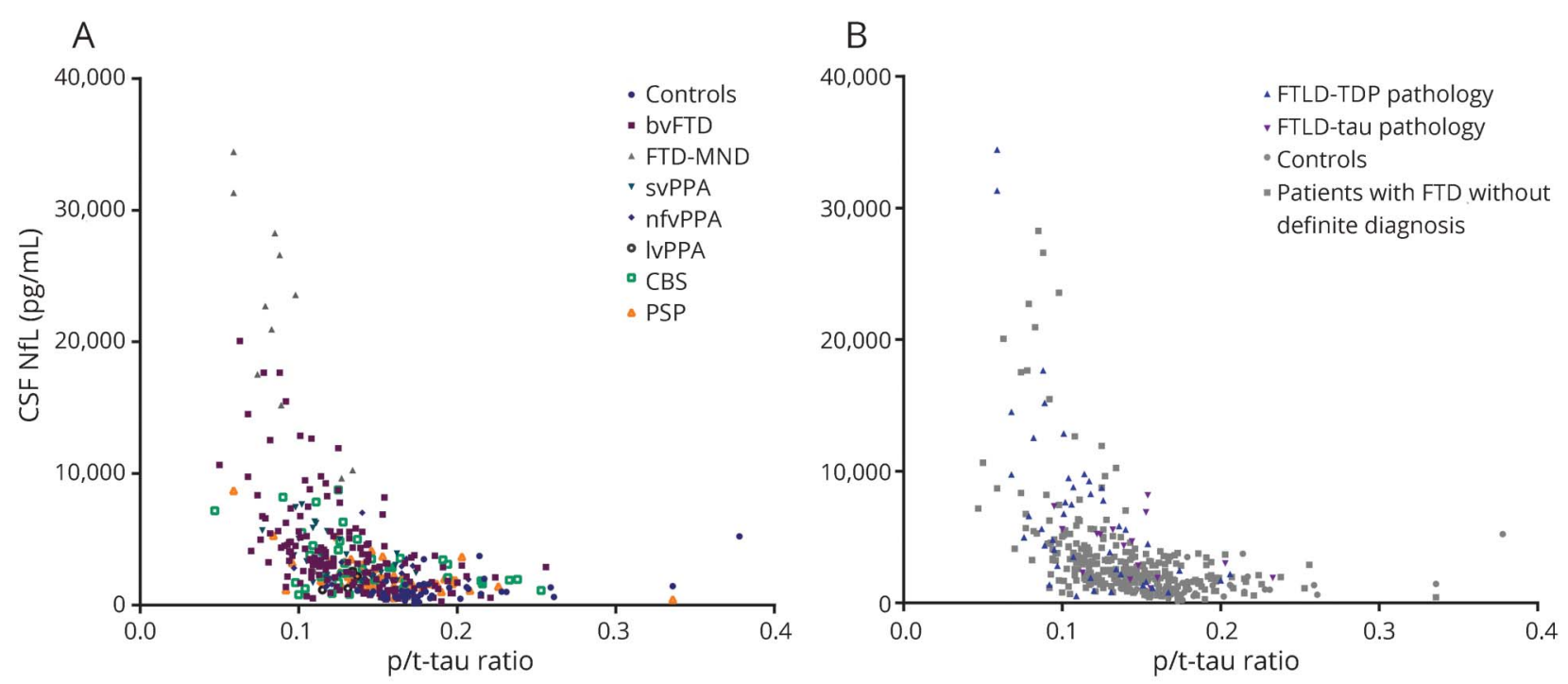

In (A) the clinical diagnoses are marked: controls (blue filled circles), bvFTD (purple filled squares), FTD-MND (gray filled upward triangles), svPPA (aqua filled downward triangles), nfvPPA (blue filled diamonds), IvPPA (black circles), CBS (green squares), and PSP (orange triangles). In (B) the association in definite diagnoses is shown: FTLD-TDP pathology (blue upward triangles), FTLD-tau pathology (purple downward triangles), controls (gray circles), and patients without a definite diagnosis (gray squares). bvFTD = behavioral variant frontotemporal dementia; CBS = corticobasal syndrome; FTD = frontotemporal dementia; FTD-MND = frontotemporal dementia with concomitant motor neuron disease; FTLD-tau = frontotemporal lobar degeneration with tau inclusions; FTLD-TDP = frontotemporal lobar degeneration with TAR DNA-binding protein 43 inclusions; IvPPA = logopenic variant primary progressive aphasia; NfL = neurofilament light chain; nfvPPA = nonfluent variant primary progressive aphasia; PSP = progressive supranuclear palsy; $\mathrm{p} / \mathrm{t}$-tau = phospho-tau/total tau; sVPPA = semantic variant primary progressive aphasia. 
Figure 3 Association of $\mathrm{NfL}$ and the p/t-tau ratio with survival
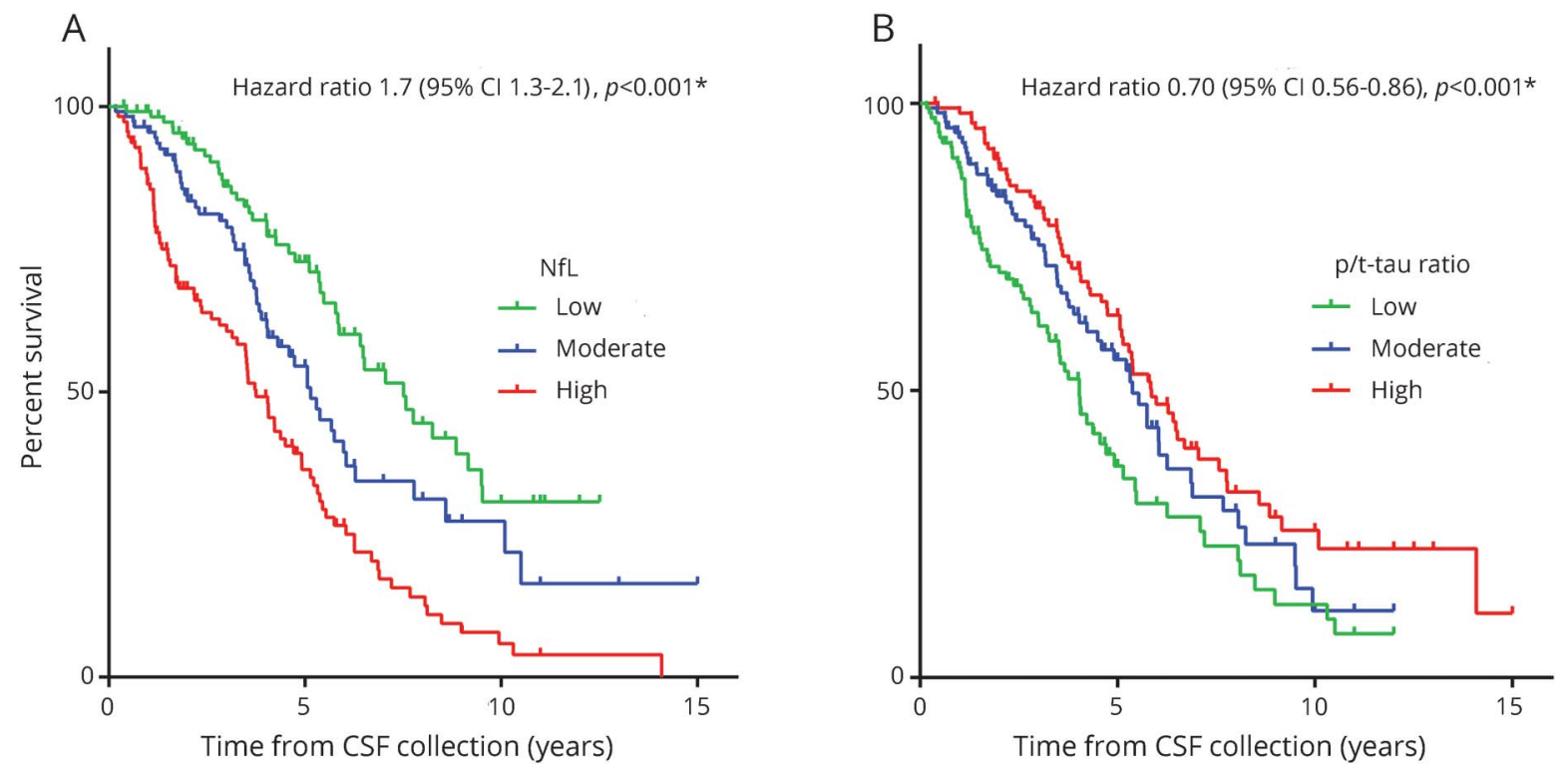

Kaplan-Meier curves of (A) NfL levels stratified to tertiles: lowest (green line; NfL <1,989 pg/mL), middle (blue line; NfL 1,989-3,675 pg/mL), or highest (red line; $\mathrm{NfL}>3,675 \mathrm{pg} / \mathrm{mL}$ ); and (B) the p/t-tau ratio stratified into lowest (green line, ratio <0.115), middle (blue line, ratio $0.115-0.146$ ), or highest (red line, ratio $>0.146$ ) tertiles. Vertical ticks represent living patients. ${ }^{*}$ Corrected for age, sex, disease duration, and motor neuron disease. $\mathrm{Cl}=$ confidence interval; $\mathrm{NfL}=$ neurofilament light chain; p/t-tau = phospho-tau/total tau.

with FTD from controls, (2) are altered in FTD-MND vs other clinical FTD subtypes, and in PSP vs bvFTD, but not between the other clinical FTD subtypes, and (3) predict survival, and that (4) $\mathrm{p} / \mathrm{t}$-tau ratio differentiates underlying TDP from tau pathology.

In clinical practice, there is a need for diagnostic markers in FTD. Prior research on NfL in CSF ${ }^{9,24-26}$ and in blood ${ }^{9,24,27}$ demonstrated a good discrimination between FTD and controls or nonneurodegenerative diseases including primary psychiatric disorders, while diagnostic performance analyses on $\mathrm{p} / \mathrm{t}$-tau ratio to discriminate patients with FTD from controls are rare. Our results show that both biomarkers, and their combination, have a good specificity to discriminate patients with clinical FTD from controls, at the drawback of a poorer sensitivity. Few false-positive results were found, but a considerable number of false negatives were found, and thus these markers support-but cannot exclude-underlying neuronal damage. Both markers are indeed involved in neuronal loss: NfL is increased and overlaps in various neurodegenerative syndromes (e.g., AD, PSP, and vascular dementia), but increases are most pronounced in FTD and amyotrophic lateral sclerosis ${ }^{7,8,28-30} ; \mathrm{p} / \mathrm{t}$-tau is decreased in various diseases characterized by marked neuronal loss, including Creutzfeldt-Jakob disease, amyotrophic lateral sclerosis, AD, and FTLD-TDP. ${ }^{10,13,14,31-33}$ Overlap in underlying pathophysiology is supported by our results showing correlation between the 2 markers.

Since both NfL and the $\mathrm{p} / \mathrm{t}$-tau ratio are aspecific neurodegeneration markers, we anticipated a limited role in subtyping different phenotypes. Indeed, NfL levels were comparable across bvFTD, nfvPPA, svPPA, and CBS, in concordance with prior results in other series. ${ }^{7,27,30,34}$ This study demonstrates a similar pattern for the $\mathrm{p} / \mathrm{t}$-tau ratio for the first time. Meanwhile, both CSF markers discriminated FTD-MND from other subtypes as supported by previous research ${ }^{10,24}$; in clinical practice, the added value of these markers may seem limited because MND is ascertained by clinical and EMG examination, but they could warn for subclinical MND. NfL and the $\mathrm{p} / \mathrm{t}$-tau ratio also differed between patients with PSP and bvFTD; nevertheless, levels considerably overlapped, yielding a diagnostic performance that is insufficient to implement in clinical practice. The lack of higher NfL and lower $\mathrm{p} / \mathrm{t}$-tau ratio in lvPPA compared with controls is likely explained by the small subgroup $(n=4)$ and not by underlying $\mathrm{AD}$ pathology because they had a definite FTD diagnosis (one C9orf72 repeat expansion, one optineurin mutation) and/or normal AD CSF biomarkers $(n=2)$.

NfL did not discriminate underlying TDP from tau pathology, or improve the performance of the ratio, which contrasts to smaller studies showing high NfL levels in FTLD-TDP. ${ }^{10,30}$ In our sample, NfL levels strongly varied in patients with $C 9$ orf 72 repeat expansions, including a considerable number of patients with low levels. Including patients with suspected pathology did yield higher NfL levels in FTLD-TDP, but this seems to result from very high levels in patients with FTD-MND. These findings underline the heterogeneity in pathophysiology in FTLD.

In concordance with earlier reports, ${ }^{10,13,14}$ we show that the $\mathrm{p} / \mathrm{t}$-tau ratio is specific to differentiate TDP from tau 
pathology, which can enable the application of diseasemodifying agents that target a specific underlying pathology. Compared with previous studies, ${ }^{13,35}$ we report different $a b$ solute tau levels and ratios because of different measurement platforms; this impedes direct comparison of the studies and illustrates the need for local cutoffs or multicenter evaluation.

The pathophysiologic background of a low $\mathrm{p} / \mathrm{t}$-tau ratio is not entirely clear; the $\mathrm{p} / \mathrm{t}$-tau ratio differed between patients and controls because of elevated t-tau levels-representing neuronal loss-in concordance with prior studies. ${ }^{10,33,36}$ In contrast, the observed differences between underlying TDP and tau pathology were mainly driven by low p-tau levels in FTLD-TDP. Prior studies have reported conflicting results: $\mathrm{p}$-tau was decreased in TDP pathology, ${ }^{13,32,35} \mathrm{t}$-tau was increased, ${ }^{14}$ or no differences were found. ${ }^{10,33}$ These differences may be explained by variation in cohort compositions, especially regarding the proportion of genetic patients, of concomitant $\mathrm{MND}$, and of $\mathrm{AD}$ copathology. We found similar $\mathrm{p} / \mathrm{t}$-tau ratios in patients with low vs high $\mathrm{AD}$ copathology, but it is not possible to draw firm conclusions since the group with copathology was too small and mostly excluded because of the study design. A recent study observed an association of antemortem CSF p-tau, and not t-tau, with postmortem cerebral tau pathology, suggesting that low p-tau levels reflect the low tau burden in FTLD-TDP. ${ }^{35}$

In the day-to-day practice, prognostic markers are important to inform patients and caregivers and to customize treatment plans. The present study corroborates the association of NfL and the $\mathrm{p} / \mathrm{t}$-tau ratio with survival, ${ }^{9,10,37}$ and shows 5-year survival rates that can be applied to clinical patients, if replicated. In clinical trials, this can aid in sample size estimations that will facilitate trial efficiency.

Lastly, these biomarkers-especially NfL_could serve as surrogate endpoints in therapeutic trials, supported by the association with disease severity we show, as in earlier reports. $^{7,9,27}$ For example, in multiple sclerosis, a dynamical decrease of CSF NfL was observed after treatment intervention. ${ }^{38} \mathrm{~A}$ similar application in dementia is endorsed by an amyloidosis mouse model, in which BACE1-inhibitor treatment reduced the $A \beta$ deposits along with CSF NfL levels. ${ }^{39}$ Furthermore, CSF NfL strongly correlates with serum NfL in FTD, ${ }^{9,24}$ implicating that NfL can be determined in a less invasive way, enabling repeated sampling. In this clinical study, we have investigated CSF, because its collection (and not serum) is an integral part of our diagnostic process to exclude $\mathrm{AD}$, and has the advantage of allowing measurement of NfL simultaneously with markers unmeasurable in blood (i.e., $\mathrm{p} / \mathrm{t}$-tau ratio). In future trial settings, one could envision a CSF measurement of the ratio and NfL at baseline, to stratify on suspected underlying pathology and disease progression, and subsequent serum NfL measurements for monitoring.

Strengths of this study include a large, well-characterized cohort with a large number of definite FTD diagnoses $(\mathrm{n}=68)$. This study was an important head-to-head comparison of $\mathrm{NfL}$ vs the $\mathrm{p} / \mathrm{t}$-tau ratio across the entire FTD spectrum, including PSP and CBS, which is representative of a memory clinic population. We also show the differences in $\mathrm{p} / \mathrm{t}$-tau ratio across clinical diagnoses. Our information can aid in moving these biomarkers from benchside to clinical practice. The exclusion of patients with a low CSF $A \beta_{42}$ and high tau may have excluded some patients with $\mathrm{FTLD}^{3}$; however, it ensures that no concomitant $\mathrm{AD}$ pathology is causing alterations in the studied biomarkers and therefore enabled a pure study cohort. A limitation is the retrospective design, which resulted in missing data and the lack of FTDspecific scales (e.g., Frontotemporal Dementia Rating Scale or FTD-CDR-SB), ${ }^{40}$ and the fact that we did not include longitudinal samples, which are necessary to determine the usability in trial settings.

Both NfL and the $\mathrm{p} / \mathrm{t}$-tau ratio thus show similar patterns in discriminating clinical FTD groups and predicting survival. This implies that the markers are interchangeable for these applications, and NfL has the advantage of being measurable in blood (although the performance in blood remains to be proven). However, to stratify underlying TDP from tau pathology, the $\mathrm{p} / \mathrm{t}$-tau ratio should be used since it outperforms NfL.

\section{Authors contributions}

L.M., E.V, A.R., W.v.d.F., C.T., J.v.S., and Y.P. designed the study. L.M. performed the statistical analyses. All authors worked on data collection, interpreting the data, and drafting or revising the manuscript.

\section{Study funding}

This study was funded by European Joint ProgrammeNeurodegenerative Disease Research and the Netherlands Organisation for Health Research and Development (RiMod-FTD 733051024), Alzheimer Nederland (L.M. [WE.09-2014-04] and C.T.), the Association of Frontotemporal Dementia (C.T.), the Dutch Research Council (ZonMW, C.T.), Weston Brain Institute (C.T.), Alzheimer's Drug Discovery Foundation (C.T.), Bluefield Project (L.D.K.), and the Dioraphte Foundation (J.v.S.). Research of the VUmc Alzheimer Center is part of the neurodegeneration research program of the Neuroscience Campus Amsterdam. The VUmc Alzheimer Center is supported by Stichting Alzheimer Nederland and Stichting VUmc fonds. Y.P. received personal funding for CSF analyses by the company IngroSyl.

\section{Disclosure}

L. Meeter, E. Vijverberg, M. Del Campo, A. Rozemuller, L. Donker Kaat, and F. de Jong report no disclosures relevant to the manuscript. W. van der Flier has performed contract research for Biogen MA Inc. and Boehringer Ingelheim, and has been an invited speaker at Boehringer Ingelheim and Biogen MA Inc. All funding is paid to her institution. C. Teunissen serves on the advisory board of Fujirebio and Roche, performed contract research for Shire, Boehringer, 
Roche, Probiodrug, PeopleBio, and Jansen Prevention Center. J. van Swieten reports no disclosures relevant to the manuscript. Y. Pijnenburg received personal funding for CSF analyses by the company IngroSyl. Go to Neurology.org/N for full disclosures.

Received August 18, 2017. Accepted in final form December 27, 2017.

\section{References}

1. Rascovsky K, Hodges JR, Knopman D, et al. Sensitivity of revised diagnostic criteria for the behavioural variant of frontotemporal dementia. Brain 2011;134(pt 9): 2456-2477.

2. Gorno-Tempini ML, Hillis AE, Weintraub $\mathrm{S}$, et al. Classification of primary progressive aphasia and its variants. Neurology 2011;76:1006-1014.

3. Meeter LH, Kaat LD, Rohrer JD, Van Swieten JC. Imaging and fluid biomarkers in frontotemporal dementia. Nat Rev Neurol 2017;13:406-419.

4. Mackenzie IRA, Neumann M, Bigio EH, et al. Nomenclature and nosology for neuropathologic subtypes of frontotemporal lobar degeneration: an update. Acta Neuropathol 2010;119:1-4.

5. Lashley T, Rohrer JD, Mead S, Revesz T. Review: an update on clinical, genetic and pathological aspects of frontotemporal lobar degenerations. Neuropathol Appl Neurobiol 2015;41:858-881.

6. Josephs KA, Hodges JR, Snowden JS, et al. Neuropathological background of phenotypical variability in frontotemporal dementia. Acta Neuropathol 2011;122: $137-153$.

7. Scherling CS, Hall T, Berisha F, et al. Cerebrospinal fluid neurofilament concentration reflects disease severity in frontotemporal degeneration. Ann Neurol 2014;75: $116-126$.

8. Skillback T, Farahmand B, Bartlett JW, et al. CSF neurofilament light differs in neurodegenerative diseases and predicts severity and survival. Neurology 2014;83: 1945-1953.

9. Meeter LH, Dopper EG, Jiskoot LC, et al. Neurofilament light chain: a biomarker for genetic frontotemporal dementia. Ann Clin Transl Neurol 2016;3:623-636.

10. Pijnenburg YAL, Verwey NA, van der Flier WM, Scheltens P, Teunissen CE. Discriminative and prognostic potential of cerebrospinal fluid phosphoTau/tau ratio and neurofilaments for frontotemporal dementia subtypes. Alzheimers Dement 2015; $1: 505-512$.

11. Steinacker P, Feneberg E, Weishaupt J, et al. Neurofilaments in the diagnosis of motoneuron diseases: a prospective study on 455 patients. J Neurol Neurosurg Psychiatry 2016;87:12-20

12. Zetterberg H, Skillbäck T, Mattsson N, et al. Association of cerebrospinal fluid neurofilament light concentration with Alzheimer disease progression. JAMA Neurol 2016;73:60-67.

13. Hu WT, Watts K, Grossman M, et al. Reduced CSF p-Tau181 to Tau ratio is a biomarker for FTLD-TDP. Neurology 2013;81:1945-1952.

14. Borroni B, Benussi A, Archetti S, et al. Csf p-tau181/tau ratio as biomarker for TDP pathology in frontotemporal dementia. Amyotroph Lateral Scler Frontotemporal Degener 2015;16:86-91.

15. Rosso SM, Kaat LD, Baks T, et al. Frontotemporal dementia in the Netherlands: patient characteristics and prevalence estimates from a population-based study. Brain 2003;126(pt 9):2016-2022.

16. van der Flier WM, Pijnenburg YAL, Prins N, et al. Optimizing patient care and research: the Amsterdam dementia cohort. J Alzheimers Dis 2014;41:313-327.

17. Litvan I, Agid Y, Calne D, et al. Clinical research criteria for the diagnosis of progressive supranuclear palsy (Steele-Richardson-Olszewski syndrome): report of the NINDS-SPSP international workshop. Neurology 1996;47:1-9.
18. Armstrong MJ, Litvan I, Lang AE, et al. Criteria for the diagnosis of corticobasal degeneration. Neurology 2013;80:496-503.

19. Dubois B, Feldman HH, Jacova C, et al. Advancing research diagnostic criteria for Alzheimer's disease: the IWG-2 criteria. Lancet Neurol 2014;13:614-629.

20. Montine TJ, Phelps CH, Beach TG, et al. National Institute on Aging-Alzheimer's Association guidelines for the neuropathologic assessment of Alzheimer's disease: a practical approach. Acta Neuropathol 2012;123:1-11.

21. del Campo M, Mollenhauer B, Bertolotto A, et al. Recommendations to standardize preanalytical confounding factors in Alzheimer's and Parkinson's disease cerebrospinal fluid biomarkers: an update. Biomark Med 2012;6:419-430.

22. Youden WJ. Index for rating diagnostic tests. Cancer 1950;3:32-35.

23. Hanley JA, McNeil BJ. A method of comparing the areas under receiver operating characteristic curves derived from the same cases. Radiology 1983;148:839-843.

24. Wilke C, Preische O, Deuschle C, et al. Neurofilament light chain in FTD is elevated not only in cerebrospinal fluid, but also in serum. J Neurol Neurosurg Psychiatry 2016;87:1270-1272.

25. Vijverberg EGB, Dols A, Krudop WA, et al. Cerebrospinal fluid biomarker examination as a tool to discriminate behavioral variant frontotemporal dementia from primary psychiatric disorders. Alzheimers Dement 2017;7:99-106.

26. Alcolea D, Vilaplana E, Suárez-Calvet M, et al. CSF sAPP $\beta$, YKL-40, and neurofilament light in frontotemporal lobar degeneration. Neurology 2017;89:178-188.

27. Rohrer JD, Woollacott IOC, Dick KM, et al. Serum neurofilament light chain protein is a measure of disease intensity in frontotemporal dementia. Neurology 2016;87: $1329-1336$.

28. Lu CH, Macdonald-Wallis C, Gray E, et al. Neurofilament light chain: a prognostic biomarker in amyotrophic lateral sclerosis. Neurology 2015;84:2247-2257.

29. Rojas JC, Karydas A, Bang J, et al. Plasma neurofilament light chain predicts progression in progressive supranuclear palsy. Ann Clin Transl Neurol 2016;3:216-225.

30. Landqvist Waldö M, Frizell Santillo A, Passant U, et al. Cerebrospinal fluid neurofilament light chain protein levels in subtypes of frontotemporal dementia. BMC Neurol 2013; 13:54.

31. Llorens F, Karch A, Golanska E, et al. Cerebrospinal fluid biomarker-based diagnosis of sporadic Creutzfeldt-Jakob disease: a validation study for previously established cutoffs. Dement Geriatr Cogn Disord 2017;43:71-80.

32. Grossman M, Elman L, McCluskey L, et al. Phosphorylated tau as a candidate biomarker for amyotrophic lateral sclerosis. JAMA Neurol 2014;71:442-448.

33. Kuiperij HB, Versleijen AAM, Beenes M, et al. Tau rather than TDP-43 proteins are potential cerebrospinal fluid biomarkers for frontotemporal lobar degeneration subtypes: a pilot study. J Alzheimers Dis 2017;55:585-595.

34. Steinacker P, Semler E, Anderl-Straub S, et al. Neurofilament as a blood marker for diagnosis and monitoring of primary progressive aphasias. Neurology 2017;88: 961-969.

35. Irwin DJ, Lleó A, Xie SX, et al. Ante mortem cerebrospinal fluid tau levels correlate with postmortem tau pathology in frontotemporal lobar degeneration. Ann Neurol 2017;82:247-258.

36. Wilke C, Deuschle C, Rattay TW, Maetzler W, Synofzik M. Total tau is increased, but phosphorylated tau not decreased, in cerebrospinal fluid in amyotrophic lateral sclerosis. Neurobiol Aging 2015;36:1072-1074.

37. Skillbäck T, Mattsson N, Blennow K, Zetterberg H. Cerebrospinal fluid neurofilament light concentration in motor neuron disease and frontotemporal dementia predicts survival. Amyotroph Lateral Scler Frontotemporal Degener 2017;18:397-403.

38. Kuhle J, Disanto G, Lorscheider J, et al. Fingolimod and CSF neurofilament light chain levels in relapsing-remitting multiple sclerosis. Neurology 2015;84:1639-1643.

39. Bacioglu M, Maia LF, Preische O, et al. Neurofilament light chain in blood and CSF as marker of disease progression in mouse models and in neurodegenerative diseases. Neuron 2016;91:56-66.

40. Boxer AL, Gold M, Huey E, et al. The advantages of frontotemporal degeneration drug development (part 2 of frontotemporal degeneration: the next therapeutic frontier). Alzheimers Dement 2013;9:189-198. 


\section{Clinical value of neurofilament and phospho-tau/tau ratio in the frontotemporal dementia spectrum}

\author{
Lieke H.H. Meeter, MD, Everard G. Vijverberg, MD, Marta Del Campo, PhD, Annemieke J.M. Rozemuller, MD, \\ Laura Donker Kaat, MD, Frank Jan de Jong, MD, Wiesje M. van der Flier, PhD, Charlotte E. Teunissen, PhD, \\ John C. van Swieten, MD, and Yolande A.L. Pijnenburg, MD
}

Correspondence Dr. Meeter

h.meeter@erasmusmc.nl

Cite as: Neurology ${ }^{\circledR}$ 2018;90:e1231-e1239. doi:10.1212/WNL.0000000000005261

\section{Study question}

Can CSF neurofilament light chain (NfL) levels and ratios of phospho-tau to total tau ( $\mathrm{p} / \mathrm{t}$-tau) distinguish frontotemporal dementia (FTD) subtypes?

\section{Summary answer}

They can distinguish patients with FTD from healthy controls (HCs) but cannot identify clinical FTD subtypes, apart from FTD with motor neuron disease (FTD-MND). The $\mathrm{p} / \mathrm{t}$-tau ratio can discriminate TDP- from tau pathology.

\section{What is known and what this paper adds}

FTD is a heterogeneous disease resulting from frontotemporal lobar degeneration (FTLD), and robust biomarkers for diagnosis and disease progression are currently unavailable. This study provides Class III evidence that $\mathrm{NfL}$ and the $\mathrm{p} / \mathrm{t}$-tau ratio are markers for the presence of FTD but not for clinical FTD subtypes.

\section{Participants and setting}

This study examined $45 \mathrm{HCs}$ and 361 patients with FTD, including behavioral variant FTD (179); FTD-MND (17); semantic variant (36), non-fluent variant (19), and logopenic variant (4) primary progressive aphasia (PPA); corticobasal syndrome (42); and progressive supranuclear palsy (PSP; 64). The patients were recruited from the Erasmus Medical Center and the VU University Medical Center based on clinical diagnoses and the availability of CSF samples. Some HCs had subjective memory complaints with normal CSF $\beta$-amyloid levels.

\section{Design, size, and duration}

Autopsies or genotyping confirmed that 18 patients had taubased pathologies (FTLD-tau) and 49 patients had TAR DNA-binding protein 43-based pathologies (FTLD-TDP). The CSF assessor, who was blinded to clinical information, quantified NfL, phosphotau, and total tau levels with ELISA. The biomarkers' diagnostic utilities were assessed with receiver operating characteristic analyses. Survival was analyzed by Cox regression models.

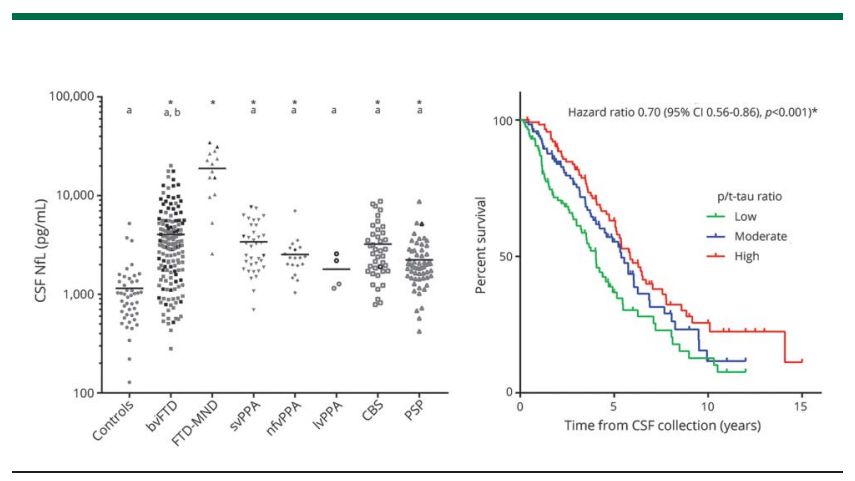

\section{Main results and the role of chance}

$\mathrm{NfL}$ levels $\geq 1,613 \mathrm{pg} / \mathrm{mL}$ and $\mathrm{p} / \mathrm{t}$-tau ratios $\leq 0.153$ both distinguished patients from HCs (NfL: sensitivity $79 \%$, specificity 89\%; area under the curve [AUC] 0.87, $p<0.001 ; \mathrm{p} / \mathrm{t}$-tau: sensitivity $73 \%$, specificity $93 \%$, AUC $0.86, p<0.001$ ); and $\mathrm{p} / \mathrm{t}$ tau ratios $\leq 0.121$ distinguished the FTLD-TDP from FTLDtau groups (sensitivity, 67\%; specificity, 76\%; AUC, 0.73; 95\% confidence interval, $0.60-0.87 ; p=0.005$ ). Both high NfL and low $\mathrm{p} / \mathrm{t}$-tau were associated with poor survival (hazard ratio on tertiles 1.7 for NfL, 0.7 for $\mathrm{p} / \mathrm{t}$-tau).

\section{Bias, confounding, and other reasons for caution}

The study was retrospective, lacked longitudinal data, and used no FTD-specific scales.

\section{Generalizability to other populations}

The exclusion of patients with possible concomitant Alzheimer disease may limit the generalizability of the results.

\section{Study funding/potential competing interests}

This study was funded by various European government agencies, medical research foundations. Dr. Pijnenburg received personal funding for CSF analyses from Ingrosyl. Dr. Teunissen reports receiving advisory committee appointments and research contracts from various pharmaceutical companies. Go to Neurology.org/ $\mathrm{N}$ for full disclosures. 


\section{Neurology}

\section{Clinical value of neurofilament and phospho-tau/tau ratio in the frontotemporal dementia spectrum}

Lieke H.H. Meeter, Everard G. Vijverberg, Marta Del Campo, et al.

Neurology 2018;90;e1231-e1239 Published Online before print March 7, 2018

DOI 10.1212/WNL.0000000000005261

\section{This information is current as of March 7, 2018}

\section{Updated Information \& Services \\ Supplementary Material}

\section{References}

Citations

Subspecialty Collections

Permissions \& Licensing

Reprints including high resolution figures, can be found at: http://n.neurology.org/content/90/14/e1231.full

Supplementary material can be found at: http://n.neurology.org/content/suppl/2018/10/01/WNL.0000000000005 261.DC1

This article cites 40 articles, 11 of which you can access for free at: http://n.neurology.org/content/90/14/e1231.full\#ref-list-1

This article has been cited by 2 HighWire-hosted articles: http://n.neurology.org/content/90/14/e1231.full\#\#otherarticles

This article, along with others on similar topics, appears in the following collection(s):

Cerebrospinal Fluid

http://n.neurology.org/cgi/collection/cerebrospinal_fluid

\section{Class III}

http://n.neurology.org/cgi/collection/class_iii

Frontotemporal dementia

http://n.neurology.org/cgi/collection/frontotemporal_dementia

Information about reproducing this article in parts (figures,tables) or in its entirety can be found online at:

http://www.neurology.org/about/about_the_journal\#permissions

Information about ordering reprints can be found online:

http://n.neurology.org/subscribers/advertise

Neurology ${ }^{\circledR}$ is the official journal of the American Academy of Neurology. Published continuously since 1951, it is now a weekly with 48 issues per year. Copyright Copyright ( 2018 The Author(s). Published by Wolters Kluwer Health, Inc. on behalf of the American Academy of Neurology. All rights reserved. Print ISSN: 0028-3878. Online ISSN: 1526-632X.

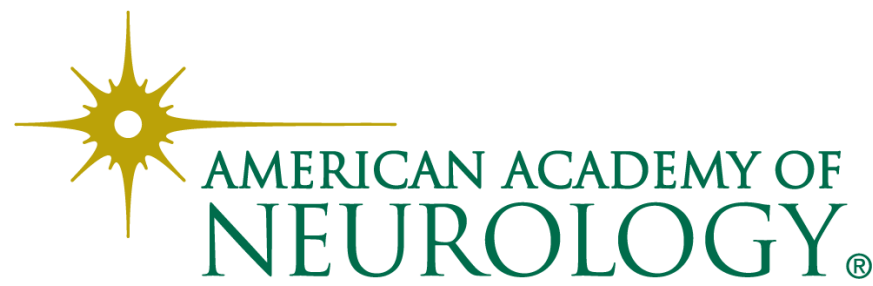

